\title{
Utility of PCT, CRP, CD64, N/L and N\% in Diagnosis of Bacterial Pneumonia in Adults: Based on the Data of 199 Bacterial Pneumonia
}

\author{
Jin Hua ${ }^{1}$, Ma Nanzhen ${ }^{2}$, Bai Haihua ${ }^{1}$, Wu Nanding ${ }^{1}$, Zhang Liping ${ }^{3}$, Liu Guoli ${ }^{1}$, \\ ${ }^{1}$ Inner Mongolia University for Nationalities, Affiliated Hospital, Nationalities, Tongliao, China \\ ${ }^{2}$ Anhui Medical University, Affiliated Hospital, Hefei, China \\ ${ }^{3}$ School of Humanistic Medicine, Anhui Medical University, Hefei, China
}

Email address:

liuguoli.888@163.com (Liu Guoli)

${ }^{*}$ Corresponding author

\section{To cite this article:}

Jin Hua, Ma Nanzhen, Bai Haihua, Wu Nanding, Zhang Liping, Liu Guoli. Utility of PCT, CRP, CD64, N/L and N\% in Diagnosis of Bacterial Pneumonia in Adults: Based on the Data of 199 Bacterial Pneumonia. International Journal of Infectious Diseases and Therapy.

Vol. 6, No. 2, 2021, pp. 69-74. doi: 10.11648/j.ijidt.20210602.15

Received: May 26, 2021; Accepted: June 8, 2021; Published: June 21, 2021

\begin{abstract}
In the background of inefficient indicators for early diagnosis of pneumonia in adults, Objective Explore the utility of PCT, CRP, CD64 and Routine Blood Parameter in diagnosis of bacterial pneumonia in adults. Methods From January 1, 2019 to December 31, 2019, 191 adult bacterial pneumonia patients diagnosed and treated in the affiliated Hospital of Inner Mongolia University for Nationalities and 99 healthy people were selected as study subjects. A total of 191 adult bacterial pneumonia patients were treated as the case group, and 99 healthy physical examination patients were treated as the control group. PCT, CRP CD64 and blood routine test parameters were compared between them. Correlation analysis and multiple linear regression analysis were used to explore the differential factors of adult bacterial pneumonia, and ROC curve was used to analyze the effectiveness of each indicator in the differential diagnosis of adult bacterial pneumonia. Results PCT (4.95 \pm 2.462 ), CRP (33.53 \pm 9.342$)$, CD64 (9.26 \pm 4.023$), \mathrm{N} / \mathrm{L}(9.54 \pm 3.878)$ and $\mathrm{N} \%(1.16 \pm 0.632)$ in the case group were significantly higher than $(0.22 \pm 0.059),(3.29 \pm 1.712),(1.91 \pm 0.600),(2.66 \pm 0.665)$ and $(0.66 \pm 0.158)$, those of the control group. There were no statistically significant differences in other indicators. Adult bacterial pneumonia was significantly correlated with PCT, CRP, CD64, N/L and N\%, but not with WBC. PCT, CRP, CD64, N/L and N\% were correlated with each other, and the correlation was statistically significant. The results of multiple linear regression model showed that PCT, CRP, CD64, N/L and N\% indexes were used for the differential diagnosis of adult bacterial pneumonia are trustworthy. The AUC $(95 \% \mathrm{CI})$ of PCT, CRP, CD64, N/L, and N\% were $0.916(0.877,0.954), 0.995(0.000,1.000), 0.980(0.962,0.997), 0.949(0.921,0.976)$, and $0.865(0.822,0.908)$, respectively. We conclude that PCT, CRP, CD64, N/L and N\% indexes are comprehensive indexes for the differential diagnosis of adult bacterial pneumonia, which have certain significance and reference value in the early identification and diagnosis of adult bacterial pneumonia.
\end{abstract}

Keywords: Bacterial Pneumonia, PCT, CRP, CD64

\section{Introduction}

Pneumonia is the inflammation of lung parenchyma caused by a variety of pathogens (such as bacteria, viruses, fungi, parasites, etc.), which is a common respiratory infectious disease in clinic. With the aggravation of air pollution and changes in living habits, pneumonia has become a high incidence disease among the population, and its incidence rate and mortality rate are increasing [1]. Pneumonia is generally divided into bacterial pneumonia, viral pneumonia, mycoplasma pneumonia, pneumonia caused by other pathogens, fungal pneumonia and pneumonia caused by other non infectious factors (such as radiation pneumonia, aspiration pneumonia, allergic pneumonia, etc.). For different pneumonia, the treatment methods and drugs are different. Therefore, early and accurate diagnosis of pneumonia is the key to effective 
treatment of pneumonia. Studies have reported that bacterial pneumonia is the most common type of pneumonia, accounting for about $80 \%$ of adult pneumonia [2]. The clinical symptoms of bacterial pneumonia in the elderly are not typical, so it is difficult to distinguish them in clinic [3]. According to epidemiological investigation, bacterial pneumonia has become the main cause of death in elderly patients [4]. Early diagnosis is directly related to curative effect. Procalcitonin (PCT) has been used in the diagnosis of bacterial infection and anti infection treatment, and achieved certain results [5]. C-reactive protein (CRP) is an acute phase reaction protein of the body [6, 7]. It has a certain clinical application value in the identification of bacterial infection, reflecting the body inflammatory infection. In 2018, García-Salido A pointed out that neutrophil index CD64 is an early sensitive indicator for the diagnosis of acute cell infection [8]. Especially when bacterial infection increased significantly, the effect was particularly obvious. Although there is not enough evidence for the diagnosis of bacterial pneumonia whether the leukocyte is elevated or not, it will produce inflammation when the cells are infected by bacteria, and the hemogram parameters will be affected by it [9] Therefore, it is suggested that the presence of parameters in the blood routine may be an effective diagnostic index of bacterial pneumonia. In 2018, Youqi et al. proposed that CRP and neutrophil count / lymphocyte count ( $\mathrm{n} / \mathrm{L}$ ) can be used for the diagnosis of bacterial pneumonia from the perspective of differential diagnosis of pneumonia types [10]. Other parameters of blood routine were not discussed in this study; CD64 has been tested in diabetic foot, severe pneumonia, puerperal infection and other bacterial infections to a certain extent [11-13], but the study of diagnosis of bacterial pneumonia in ordinary adults has not been carried out. In 2020, Zhan Wenli explored the diagnostic value of CD64, CRP, PCT and NLR in children with community-acquired pneumonia. However, there are differences in immunity between adults and children, and the utility of CD64, CRP, PCT and NLR in the diagnosis of adult bacterial pneumonia needs to be further verified [14]. Combined with the fact that the diagnostic accuracy of single index is not high, the comprehensive diagnostic research of PCT, CRP, CD64 and blood routine is carried out to form a comprehensive index for the early diagnosis of adult bacterial pneumonia, so as to provide scientific basis for symptomatic treatment and scientific medication of adult bacterial pneumonia patients, and improve the quality of life of patients.

\section{Methods}

\subsection{Data Colletion}

191 patients with bacterial pneumonia diagnosed and treated in the outpatient and inpatient department of Affiliated Hospital of Inner Mongolia University for Nationalities from January 1, 2019 to December 31, 2019 were selected as the case group, including 90 males and 101 females, aged from 18 to 93 years. In the same period, 100 physical examinees were randomly selected from the physical examination center, of which 99 healthy examinees were set as the normal control group, including 46 males and 53 females, aged from 19 to 79 . The patients in this study have signed the informed consent and the study is approved by the medical ethics committee of the Affiliated Hospital of Inner Mongolia University for nationalities.

\subsection{Instruments and Reagents}

Analyzed by xn9000 hematology analyzer (Japan XISEN Meikang company) and 2-3ml EDTA anticoagulant was collected. The leukocytes were counted by flow cytometry and nucleic acid fluorescence staining. C-reactive protein was detected by turbidimetry; Cobas 8000e602 Luminescent Immunoassay Analyzer was used for procalcitonin detection, and BD FACSAria II flow cytometry was used for neutrophil CD64 index detection.

\subsection{Inclusion and Exclusion Criteria}

Inclusion Criteria, First, bacterial pneumonia meets the diagnostic criteria of Chinese Guidelines for the Diagnosis and Treatment of Community-Acquired Pneumonia for Adults (2016 Edition). Second, all subjects had not received any medication before blood sample collection.

Exclusion criteria, First, there was a history of treatment before blood samples were collected. Second, lack of important data. Third, Except for pulmonary tumor, pulmonary tuberculosis, non infectious pulmonary interstitial disease, pulmonary edema, atelectasis, pulmonary embolism, pulmonary eosinophilic infiltration and pulmonary vascular disease.

\subsection{Measures Used}

Using SPSS 19.0, a descriptive analysis was done for the measurement data (mean, standard deviation). T test was used for the mean between the two groups. Pearson correlation coefficient analysis was used to analyze the correlation of PCT, CRP, CD64 and blood routine test parameters. Multivariate logistic regression analysis was used to explore the early diagnosis indexes of bacterial pneumonia, and the receiver operating characteristic curve (ROC) was drawn. Differences are considered to be statistically significant when $\mathrm{P}<0.05$.

\section{Results}

\subsection{Comparison of Clinical Indexes Between Case Group and Control Group}

In adult bacterial pneumonia, there was no significant difference in gender and age between case group and control group $(\mathrm{P}>0.05)$. There were significant differences in PCT, CRP, CD64, N/L, WBC, N\%, L\% and M\% $(\mathrm{P}<0.05)$. There was no significant difference between $\mathrm{L} \%$ and $\mathrm{M} \%(\mathrm{P}>0.05)$ (see Table 1). 
Table 1. Comparison of each index level between case group and control group $(\mu \pm s)$.

\begin{tabular}{llll}
\hline index & Case group $(\mathbf{n = 1 9 1 )}$ & Control group $(\mathbf{n = 9 9 )}$ & $\boldsymbol{T}$ \\
\hline Gender & $1.53 \pm 0.501$ & $1.54 \pm 0.501$ & 0.114 \\
Age & $56.94 \pm 14.676$ & $59.68 \pm 12.419$ & 3.760 \\
PCT & $4.95 \pm 2.462$ & $0.22 \pm 0.059$ & 134.084 \\
CRP & $33.53 \pm 9.342$ & $3.29 \pm 1.712$ & 129.543 \\
CD64 & $9.26 \pm 4.023$ & $1.91 \pm 0.600$ & 24.789 \\
N/L & $9.54 \pm 3.878$ & $2.66 \pm 0.665$ & 85.903 \\
WBC & $7.85 \pm 5.224$ & $8.52 \pm 4.667$ & 0.053 \\
N\% & $1.16 \pm 0.632$ & $0.66 \pm 0.158$ & 0.000 \\
L\% & $0.14 \pm 0.082$ & $0.24 \pm 0.077$ & 0.000 \\
M\% & $0.07 \pm 0.056$ & $0.08 \pm 0.033$ & 0.000 \\
\hline
\end{tabular}

\subsection{Correlation Analysis}

There was significant correlation between PCT, CRP, CD64, $\mathrm{N} / \mathrm{L}$ and $\mathrm{N} \%$ and adult bacterial pneumonia, and there was statistical significance $(\mathrm{P}<0.05)$. There was no significant correlation with gender, age and WBC $(\mathrm{P}>0.05)$. PCT, CRP,
CD64, N/L and N\% were correlated with each other $(\mathrm{P}<0.05)$. The correlation coefficients of PCT with CRP and N/L were 0.740 and 0.799 , respectively. The correlation coefficients of CRP with CD64 and N/L were 0.653 and 0.735 , respectively (see Table 2).

Table 2. Correlation between indexes.

\begin{tabular}{|c|c|c|c|c|c|c|c|c|c|}
\hline index & 1 & 2 & 3 & 4 & 5 & 6 & 7 & 8 & 9 \\
\hline 1. Groups & 1 & & & & & & & & \\
\hline 2. Gender & -.009 & 1 & & & & & & & \\
\hline 3. Age & -.091 & $-.144 *$ & 1 & & & & & & \\
\hline 4. РCT & $.731 * * *$ & -.038 & -.011 & 1 & & & & & \\
\hline 5. CRP & $.794 * * *$ & -.034 & -.017 & $.740 * * *$ & 1 & & & & \\
\hline 6. CD64 & $.715 * * *$ & -.086 & -.051 & $.515 * * *$ & $.653 * * *$ & 1 & & & \\
\hline 7. N/L & $.704 * * *$ & -.008 & -.026 & $.799 * * *$ & $.735 * * *$ & $.513 * * *$ & 1 & & \\
\hline 9. N\% & $.407 * * *$ & -.009 & -.062 & $.473 * * *$ & $.299 * * *$ & $.236 * * *$ & $.334 *$ & $-.424 * * *$ & 1 \\
\hline
\end{tabular}

Notes: Groups, Grouping of case health status. ${ }^{*},<0.05 ; * *,<0.01 ; * * *,<0.001$.

Factor collinearity test showed that PCT, CRP, CD64 and $\mathrm{N} / \mathrm{L}$ played an independent role in the early clinical diagnosis of adult bacterial pneumonia, so there was no collinearity problem in our study [15-17], see Table 3.

Table 3. Factor covariance test.

\begin{tabular}{|c|c|c|c|c|}
\hline index & $B$ & $T$ & $P$ & VIF \\
\hline PCT & 0.025 & 2.954 & 0.003 & 3.700 \\
\hline CRP & 0.009 & 6.805 & 0.000 & 3.153 \\
\hline CD64 & 0.031 & 8.443 & 0.000 & 1.756 \\
\hline $\mathrm{N} / \mathrm{L}$ & 0.012 & 2.386 & 0.018 & 3.184 \\
\hline N\% & 0.090 & 3.448 & 0.001 & 1.304 \\
\hline
\end{tabular}

\subsection{Multiple Linear Linear Regression Model}

Based on the above research, we further take whether the sample is adult bacterial pneumonia as the dependent variable (binary variable), and take PCT, CRP, CD64, N/L and N\% as independent variables respectively to do multiple linear regression analysis. The results are shown in Table 4.

Table 4. Diagnostic model for each indicator of adult bacterial pneumonia.

\begin{tabular}{|c|c|c|c|c|}
\hline Variables & Model 1 & Model 2 & Model 3 & Model 4 \\
\hline $\mathrm{C}$ & -0.015 & 0.051 & 0.017 & $0.078 * *$ \\
\hline PCT & $0.025 * *$ & $0.036 * * *$ & $0.036 * * *$ & $0.045 * * *$ \\
\hline CRP & $0.009 * * *$ & $0.009 * * *$ & $0.010 * * *$ & $0.010 * * *$ \\
\hline CD64 & $0.031 * * *$ & $0.031 * * *$ & $0.031 * * *$ & $0.032 * * *$ \\
\hline $\mathrm{N} / \mathrm{L}$ & $0.012 *$ & $0.011 *$ & & \\
\hline N\% & $0.009 * *$ & & $0.086 * *$ & \\
\hline$R^{2}$ & 0.750 & 0.741 & 0.746 & 0.737 \\
\hline$F$-statistic & $185.009 * * *$ & $220.518 * * *$ & $226.40 * * *$ & $289.086^{* * * *}$ \\
\hline
\end{tabular}

Notes: $\mathrm{n}=290,{ }^{*} \mathrm{p}<0.01 ;{ }^{* *} \mathrm{p}<0.05,{ }^{* * *} \mathrm{p}<0.001$ 
It can be seen from the above results that the parameters of the above four models show the $\mathrm{R}^{2}$ of model 1 is 0.750 , which are greater than the $\mathrm{R}^{2}$ of other models. The adj values of $\mathrm{R}^{2}$ of model 1 were higher than those of other models, and the degree of approach was higher than that of model 2 and model 3 , which was equivalent to that of model 4 . Therefore, it can be concluded that the diagnostic utility of model 1 is most consistent with the actual diagnostic data, and model 1 has good reliability.

\subsection{Analysis of Clinical Indicators Utility in Diagnostic}

The AUC of PCT, CRP, CD64, N / L and N\% in the diagnosis of adult bacterial pneumonia were $0.916,0.995$, $0.980,0.949$ and 0.865 respectively, which were all satisfactory predictors, AUC $>0.7$ was the standard for satisfactory prediction [18]. The detailed data are shown in figure 1 .

\section{ROC Curve}

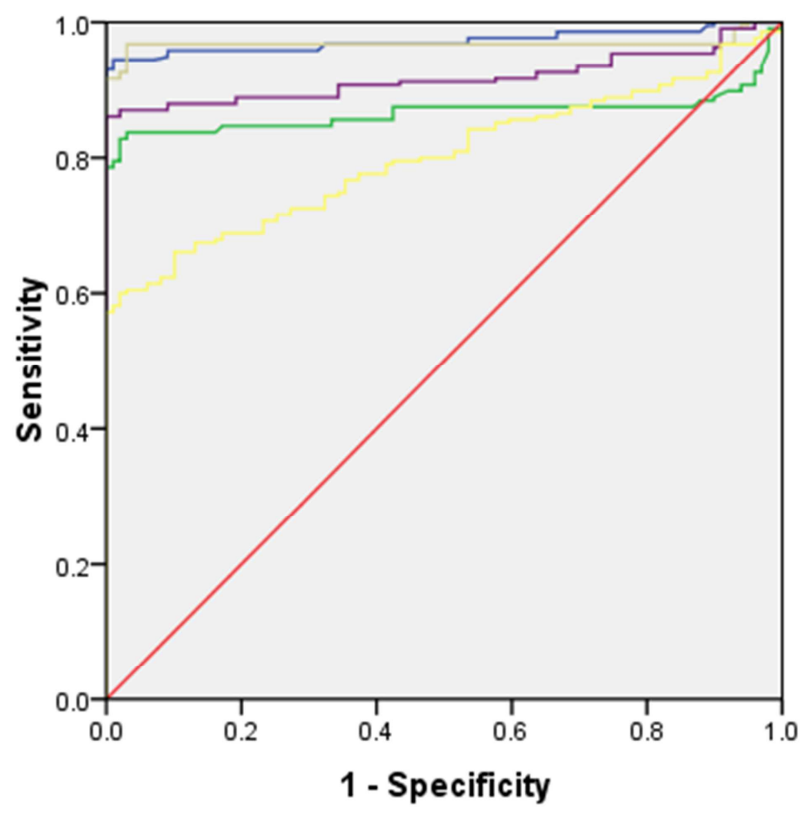

Source of the Curve

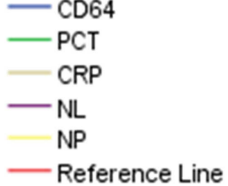

Notes: NLfor N/L; NP for N\%.

Figure 1. ROC of PCT 、CRP、CD64、N/L and N\% levels for adult bacterial pneumonia.

\section{Discussion}

Bacterial pneumonia is one of the diseases leading to pulmonary infection, and pulmonary infection has become an important cause of hospitalization and death [19]. Timely and accurate diagnosis of the type of pneumonia and grasp the degree of illness are the primary condition of effective treatment and prognosis of bacterial pneumonia. Therefore, it is of theoretical significance and practical value to explore the scientific and efficient diagnostic indicators of bacterial pneumonia.

Neutrophil CD64 is an IgG mediated Fc receptor with high affinity for immunoglobulin IgG. It is mainly distributed on the surface of macrophages, monocytes and dendritic cells [20]. Under normal conditions, only a small amount of CD64 is expressed on the surface of neutrophils, and the expression will be rapidly up-regulated in bacterial infection. The results showed that the correlation coefficient between adult bacterial pneumonia and CD64 expression was 0.715, and the AUC (95\% CI) of CD64 in the diagnosis of bacterial pneumonia was 0.980 $(0.962,0.997)$. It can be seen from both of them that CD64 can be used as an effective index for early diagnosis of adult bacterial pneumonia. This is consistent with the results of literature [21, 22]. According to the analysis of multiple linear regression model, it is the best choice to comprehensively use PCT, CRP, CD64, $\mathrm{N} / \mathrm{L}$ and $\mathrm{N} \%$ as the comprehensive indicators in the differential diagnosis of adult bacterial pneumonia. It has certain clinical significance and practical value in the early identification and diagnosis of adult bacterial pneumonia.

The results of this study showed that the levels of PCT, CRP, $\mathrm{N} / \mathrm{L}$ and $\mathrm{N} \%$ in the case group of adult bacterial pneumonia were significantly higher than those in the control group, and they were significantly correlated with adult bacterial pneumonia. Therefore, PCT, CRP, N/L and N\% can be used as the reference standard for early diagnosis of adult bacterial pneumonia. To a certain extent, this result is consistent with the results of literature [14], and expands the results of literature [14] at the same time.

The results of the analysis of diagnostic effectiveness of clinical indicators by ROC curve showed that AUC (95\% CI) of CRP in bacterial pneumonia was $0.995(0.000,1.000)$, which can be found that CRP index is extremely sensitive and should be cautious when applied. PCT reflects the active 
degree of systemic inflammatory response. In normal metabolism, thyroid $\mathrm{C}$ cells secrete and produce calcitonin with hormone activity. The consistence of PCT in healthy individuals is low $(0.22 \pm 0.059)$, accompanied by the increase of PCT in bacterial infection (4.95 \pm 2.462$)$. Further research shows that the AUC $(95 \% \mathrm{CI})$ of PCT in the diagnosis of bacterial pneumonia was $0.916(0.877,0.954)$, indicating that PCT, as a glycoprotein secreted by thyroid gland, is highly expressed in adult patients with bacterial pneumonia, which is a good early differential indicator. This is consistent with the results of literature [23].

\section{Conclusion}

The levels of PCT, CRP, N/L and N\% in the case group of adult bacterial pneumonia were significantly higher than those in the control group. There were significant differences in PCT, CRP, CD64, N / L, N\%. PCT, CRP, CD64, N/L and N\% were positively correlated in adults with bacterial pneumonia, which revealed that PCT, CRP, CD64, N/L and N\% could provide evidence for the diagnosis and identification of adult bacterial pneumonia. Based on the significant correlation and statistical significance of each index, the index combined detection of PCT, CRP, CD64, N/L and N\% is helpful to improve the reliability of early diagnosis of adult bacterial pneumonia, it is the best choice to comprehensively use PCT, CRP, CD64, N/L and N\%, has certain reference value for clinical symptomatic treatment and rational drug use, and lays a theoretical foundation for improving the quality of life of patients.

This paper has certain shortcomings, for example, the difference in diagnostic utility between models is not significant enough. One possible reason for this comes from the small amount of data.

\section{Conflicts of Interest}

All the authors do not have any possible conflicts of interest.

\section{Acknowledgements}

This work was supported in part by the Natural Science Foundation of China under Grant No. 81960166, Natural Science Foundation of Inner Mongolia University For Nationalities under Grant No. NMDYB18084, NMDYB18099, Natural Science Foundation for the Higher Education Institutions of Anhui Province of China (KJ2019A0945), and Projects for policy consultation of Health Development Strategy Research Center in Anhui Province (2021szk004).

\section{References}

[1] Zuo B., Liu X., Cui W., et al. (2020) Chandes of serum adiponectin and leptin in elderly patients with bacterial pneumonia, Chin J Nosocomiol, 4 (30): 512-516.
[2] CaoJ., Li Q., Cheng R. (2019) Clinical evaluation of combined detection of serum procalcitonin and C-reactive protein for bacterial pneumonia in adults, Chin J Nosocomiol, 24 (19): 4933-4935.

[3] Jelcic I., Jelcic I., Kempf C., et al. Mechanisms of immune es-cape in central nervous system infection with neurotropic JC virus variant [J]. Annals of Neurology, 2016, 79 (3): 404-418.

[4] Chandorkar G., Xiao A., Mouksassi S., et al. Population pharmacokinetics of ceftolozane/tazobactam in healthy volunteers, subjects with varying degrees of renal function and patients with bacterial infections [J]. Journal of Clinical Pharmacology, 2015, 55 (2): 230-239.

[5] Sun S., Wang F., Yu M., et al. Clinical study of serum procalcitonin level in patients with myocardial infarction complicated by pulmonary infection [J]. Experimental \& Therapeutic Medicine, 2018, 16 (6): 5210-5214.

[6] Haruki K., Shiba H., Horiuchi T., et al. Impact of the C-reactive protein to albumin ratio on long-term outcomes after hepatic resection for colorectal liver metastases [J]. American Journal of Surgery, 2017, 214 (4): 752-756.

[7] Hu L., Shi Q., Shi M., et al. Diagnostic Value of PCT and CRP for Detecting Serious Bacterial Infections in Patients With Fever of Unknown Origin: A Systematic Review and Meta-analysis [J]. Applied Immunohistochemistry \& Molecular Morphology Aimm, 2017, 25 (8): e61-e69.

[8] García-Salido A., Serrano-González A., Casado-Flores J., et al. CD64 on monocytes and granulocytes in severe acute bronchiolitis: Pilot study on its usefulness as a bacterial infection biomarker [J]. Journal of Leukocyte Biology, 2018, 103 (5): 965-971.

[9] Principi, Nicola, Esposito, et al. Biomarkers in Pediatric Community-Acquired Pneumonia [J]. International Journal of Molecular Sciences, 2017, 18 (2): E447.

[10] You, Q., Huang, Z. M., Guo, H., Chen, J., \& Ji, L. I.. (2018). Value of inflammatory indexes and blood routine in differential diagnosis of viral and bacterial pneumonia patients and in prediction of complications. Chinese Journal of Nosocomiology, 28 (9): 1342-1351.

[11] Wang J., Zhao Z., Li F. (2020). Assessment and prognostic value of inflammatory factors, D-dimer, CD64and fibrinogen in patients with diabetic foot infection. Chinese Journal of Nosocomiology, 30 (3): 373-377.

[12] Zhang, J., Lan, L. I., \& Fan, S. M.. (2019). Diagnostic and prognostic values of CD64 index, STREM-1 and CRP/Alb ratio in patients with severe pneumonia. Journal of Tropical Medicine, 19 (7): 890-893.

[13] Zhang Q., Bian J., Lin A. (2019). Clinical diagnositic value of neutrophil CD64 for development of maternal puerperal infections. Chinese Journal of General Practice, 17 (10): 1705-1707.

[14] Zhan W., Su X. (2020). Diagnostic value of CD64, CRP, PCT and NLR in children with community acquired pneumonia. Journal of Pulmonology Clinical, 25 (5): 701-706.

[15] Sun, J., Zhang, L., Sun, R., et al. Exploring the influence of resiliency on physician trust in patients: An empirical study of Chinese incidents [J]. PLoS ONE, 2018, 13 (12): e0207394. https://doi.org/10.1371/journal.pone.0207394. 
[16] Sun J., Wang P., Du Y., et al. Analysising the influence factors of single task pricing based on public packet system: An Empirical Study in China [J]. Journal of Physics. Conference series. 2020, 1437, 012100: 1-6.

[17] Sun J., Sun R., Jiang Y., et al. The relationship between psychological health and social support: Evidence from physicians in China [J]. PLoS ONE, 2020, 15 (1): e0228152. https://doi.org/10.1371/journal.pone.0228152.

[18] Wang X., Lan H. (2019) Expression characteristics and clinical significance of TREM-1 and CD64 on the surface of umbilical cord blood mononuclear cells and neutrophils in newborns with premature rupture of membranes. Maternal and Child Health Care of China, 34 (18): 4219-4222.

[19] Viitanen S., Lappalainen A., Koho N., et al. Recurrent bacterial pneumonia in Irish Wolfhounds: Clinical findings and etiological studies [J]. Journal of Veterinary Internal Medicine, 2019, 32 (2): 846-855.
[20] Chaiyasit N., Romero R., Chaemsaithong P., et al. Clinical chorioamnionitis at term VIII: a rapid MMP -8 test for the identification of intra -amniotic inflammation [J]. Journal of Perinatal Medcine, 2017, 45 (5): 539-550.

[21] Dai J., Jiang W., Min Z., et al. Neutrophil CD64 as a diagnostic marker for neonatal sepsis: Meta-analysis [J]. Advances in Clinical and Experimental Medicine, 2017, 26 (2): 327-332.

[22] Rogina P., Stubljar D., Lejko Z., et al. Neutrophil CD64 molecule expression can predict bloodstream infection in septic shock patients [J]. Clinical Chemistry \& Laboratory Medicine, 2017, 55 (6): e130-e132.

[23] Vatan A., Saltoglu N., Yemisen M., et al. Association between biofilm and multi/extensive drug resistance in diabetic foot infection [J]. International Journal of Clinical Practice, 2018, 72 (3): e13060. 